\title{
Evaluation of endovascular abdominal aortic aneurysm repair in nonagenarians
}

\author{
H.P. Zhang, W. Guo, X.P. Liu, X. Jia, J. Xiong, X.H. Ma, M.H. Zhang \\ and Y.L. Xu \\ Department of Vascular Surgery, Chinese PLA General Hospital, Haidian, \\ Beijing, China \\ Corresponding author: W. Guo \\ E-mail: weiguochn@yeah.net
}

Genet. Mol. Res. 12 (4): 6907-6914 (2013)

Received March 1, 2013

Accepted July 31, 2013

Published December 19, 2013

DOI http://dx.doi.org/10.4238/2013.December.19.9

\begin{abstract}
The aim of this study was to investigate the safety and efficacy of endovascular abdominal aortic aneurysm repair (EVAR) in nonagenarians. From May 2003 to March 2011, 12 nonagenarian abdominal aortic aneurysm patients were treated with endovascular repair including two emergencies. The mean age of patients was 92.5 \pm 1.6 years (range: $90-95$ years), and 11 of the patients (91.7\%) were men. Technical success, perioperative complications, and mortality were evaluated. The follow-up protocol consisted of computed tomography angiograms or ultrasounds performed at 3, 6, 9, and 12 months, and annually thereafter. Seven patients were operated under general anesthesia and five under local anesthesia. There was $100 \%$ technical success with no need for open conversion. The endografts used included two Endurant endografts, four Talent endografts, and six Zenith endografts. The mean operative time was $3.4 \pm 1.3 \mathrm{~h}$, mean procedural blood loss was $150.5 \pm 60.5 \mathrm{~mL}$, and mean postoperative length of stay was $8.4 \pm 2.3$ days. Mortality rates were $8.3 \%$ at 30 days, $16.7 \%$ at 1 year, $41.7 \%$ at 3 years, and $75 \%$ at 5 years. The mean survival of the 11 patients who expired beyond the first 30 days was 28.5 months (range: 9-73 months). Overall, EVAR in nonagenarians
\end{abstract}


was associated with acceptable procedural success and perioperative morbidity and mortality. The medium and long-term results suggested that EVAR may be of limited benefit in some patients who are aged $>90$ years. Therefore, individual patient selection is very important.

Key words: Abdominal aorta; Aneurysm; Stent; Endovascular repair; Nonagenarian

\section{INTRODUCTION}

The Chinese population census of 2011 revealed that the number of people 65 years and older was $118,830,000$, accounting for $8.9 \%$ of the total population. The United States Census Bureau stated that in the next few decades, the number of people 85 years and older is expected to increase by 3 -fold (U.S. Census Bureau, 2009). Age is an important and dangerous factor of abdominal aortic aneurysms (AAA), which is the most common aortic disease among the elderly population. With the continuous increase of the elderly population, the incidence and diagnostic rate of AAA is expected to increase.

The fundamental principle behind elective treatment of AAA is to prevent future rupture and its associated morbidity and mortality. However, this must be carefully weighed against the inherent risks associated with operative intervention. Due to age-related and multiorgan complications of nonagenarians, it is widely accepted that open operations should not even be considered in high-risk patients aged 90 and above (Lange et al., 2005). With respect to AAA patients in good anatomical condition, endovascular aortic repair (EVAR) has become the first choice in place of traditional open operations. Although several studies have revealed that the mortality rate and incidence of complications in patients aged 80 years and older during the operation period and in the medium to long term were higher than those of younger patients, almost all authors have indicated that if preoperative evaluations are conducted, the results of EVAR technology would nonetheless be satisfactory (Brinkman et al., 2004; Haug et al., 2005; Henebiens et al., 2008). Therefore, for symptomatic AAA patients aged 90 or older, decision-makers should primarily focus on the risk of repair and on subjective issues related to life expectancy.

Some recent studies have reported results of applying EVAR technology to patients aged 90 and older. The number of such cases is limited due to the general lack of nonagenarian patients. Consequently, a random control study over a large range cannot be conducted. Thus, there is currently no data effectively comparing the success of EVAR in the nonagenarian population in China. Therefore, we used the data of our center to evaluate the safety and validity of conducting EVAR on such patients.

\section{MATERIAL AND METHODS}

\section{General material}

From May 2003 to March 2011, the EVAR procedure was performed on 12 AAA patients aged 90 years or older, 10 of which underwent selective operations, and two underwent emergency operations, one with a ruptured AAA, and the other with impending rupture. The 
mean age of patients was $92.5 \pm 1.6$ years (range: $90-95$ years). Eleven of the patients were males $(91.7 \%)$. Ten of the patients presented combined coronary heart disease $(83.3 \%)$, nine hypertension, and five lipid metabolic disease (33.3\%). Four patients had atherosclerotic peripheral artery occlusive disease (33.3\%), four had cancer (33.3\%), and three had chronic obstructive pulmonary disease (25\%). Two suffered chronic renal insufficiency $(16.7 \%)$ and five had a history of smoking (41.7\%). According to American Society of Anesthesiologists (ASA) scores, two cases were type IV, eight cases were type III, and two cases were type II. Seven cases had a history of abdominal operations (resection of gastric and colon cancer, gallbladder, or appendix) (Table 1). All patients had a computed tomography (CT) angiography test.

\begin{tabular}{|c|c|c|c|c|c|c|}
\hline \multirow[t]{2}{*}{ Patient } & \multirow[t]{2}{*}{ Age (years) } & \multirow[t]{2}{*}{ Comorbidities and high risk factors } & \multicolumn{3}{|c|}{ AAA characteristics } & \multirow{2}{*}{$\begin{array}{l}\text { Diameter of } \\
\text { CFA (mm) }\end{array}$} \\
\hline & & & Diameter (mm) & Neck length (mm) & Infrarenal angle $\left(^{\circ}\right)$ & \\
\hline 1 & 91 & CAD, PAOD, HT, LMD & 55 & 20 & 15 & 12 \\
\hline 2 & 95 & CAD, HT, Cancer, PAS & 67 & 25 & 45 & 7 \\
\hline 3 & 93 & CAD, HT, COPD, LMD & 73 & 16 & 60 & 8 \\
\hline 4 & 92 & CAD, HT, COPD, CRI & 76 & 18 & 15 & 10 \\
\hline 5 & 93 & CAD, HT, Cancer, PAS & 68 & 22 & 20 & 11 \\
\hline 6 & 90 & CAD, PAOD, HT, CRI & 62 & 30 & 0 & 9 \\
\hline 7 & 91 & COPD, PAS, PAOD & 85 & 25 & 30 & 8 \\
\hline 8 & 92 & CAD, HT, PAS & 57 & 10 & 0 & 6 \\
\hline 9 & 95 & CAD, HT, Cancer, PAS & 55 & 14 & 30 & 9 \\
\hline 10 & 93 & CAD, LMD, PAOD, HT & 70 & 15 & 50 & 11 \\
\hline 11 & 94 & LMD, Cancer, PAS & 75 & 19 & 25 & 10 \\
\hline 12 & 91 & CAD, HT, LMD, PAS & 65 & 18 & 40 & 6 \\
\hline Average & $92.5 \pm 1.6$ & & $67.3 \pm 9.2$ & $19.3 \pm 5.5$ & $27.5 \pm 18.9$ & $8.9 \pm 2.0$ \\
\hline
\end{tabular}

Diameter of AAA = maximum transverse diameter; neck length = length between the lowest renal artery and the point of the initial aneurysm dilatation; infrarenal angle = angle between the axes of neck and AAA; CAD $=$ coronary artery disease $; \mathrm{HT}=$ hypertension $; \mathrm{COPD}=$ chronic obstructive pulmonary diseases; $\mathrm{CRI}=$ chronic renal insufficiency; PAS = previous abdominal surgery; $\mathrm{LMD}=$ lipid metabolic disease; $\mathrm{PAOD}=$ peripheral artery occlusive disease.

\section{Methods and instruments}

The repair was performed with local or general anesthesia. Patients suffering from renal insufficiency were treated with hydration $12 \mathrm{~h}$ before and after the repair with 1-1.5 $\mathrm{mL} / \mathrm{kg}$ saline. Four approaches were taken for stent-graft delivery system access: exposure of bilateral common femoral arteries (CFA), unilateral CFA exposure and contralateral puncture, and bilateral CFA puncture. The "preclose" technique was used in the puncture position, namely using one ProGlide (Abbott, US) presetting for delivery systems under $16 \mathrm{~F}$, and two for those over $16 \mathrm{~F}$. The operation methods were conducted in line with Dosluoglu et al. (2007). Endovascular stent grafts were used on all patients, including the Zenith (Cook, US), Talent (Medtronic, US), and Endurant (Medtronic, US) grafts. The maximum diameters of the main delivery systems were $22 \mathrm{~F}$ (inner diameter), $24 \mathrm{~F}$ (external diameter), and $20 \mathrm{~F}$ (external diameter), respectively. The diameters of iliac stent grafts ranged from 14 to $18 \mathrm{~F}$. The diameter of the Endurant delivery system was smaller than those of the other two. After the repair, endoleaks were observed in the angiography. Balloon dilatation was performed conditionally. Finally, the incisions were sutured. 


\section{Statistical analysis and follow-up}

Technical success was identified according to the Society for Vascular Surgery/ American Association for Vascular Surgery (SVS/AAVS) 2002 reporting standards: successful deployment of the stent graft with no need for open conversion, no death, no evidence of type I or type III endoleaks, and no occlusion within the stents in the first $24 \mathrm{~h}$ after repair. The success of the "preclose" suture was defined as a good healing puncture point within 30 days after repair with no need for secondary treatment. Any comorbidity, such as bleeding or wound infection, associated with the puncture point was considered as a technical failure, regardless of whether an operation was needed. Clinical success was defined as successful deployment without death because of aneurysm-related treatment, freedom from type I or type III endoleaks, no aneurysm expansion, rupture, graft failure, graft migration, or conversion to open repair. Aneurysm-related death included any death resulting from aneurysm rupture, a primary or secondary endovascular procedure, or surgical conversion. Success was also considered when there was no aneurysm expansion in type II endoleaks. The parameters of clinical assessment included the operation time, amount of bleeding, days in hospital, and time in intensive care unit (ICU). The CHISS software system was used to analyze the data, and $\mathrm{P}<$ 0.05 indicated statistical significance.

The follow-up protocol consisted of CT angiography and Doppler ultrasounds to observe changes in aneurysm diameter, endoleaks, sac thrombus, iliac artery, the blood supply of lower extremity arteries, the stent patency, and whether there were expansions of anchoring vessel areas, which were performed at 3, 6, 9, and 12 months, and annually thereafter. Particular attention was paid to intestinal ischemia and gluteal ischemia, as well as to whether there was a combination of dissecting, stenosis, pseudoaneurysm, or arteriovenous fistula at the CFA.

\section{RESULTS}

\section{AAA characteristics and technical aspects}

The average diameter of infrarenal AAA in the 12 cases was $67.3 \pm 9.2 \mathrm{~mm}$ (range: 55-85 mm). The mean neck length of AAA was $19.3 \pm 5.5 \mathrm{~mm}$ (range: $10-30 \mathrm{~mm}$ ). The mean infrarenal angle was $27.5 \pm 18.9^{\circ}$ (range: $0-60^{\circ}$ ). The mean diameter of CFA was $8.9 \pm 2.0 \mathrm{~mm}$ (range: 6-12 mm). Three cases presented unilateral common iliac aneurysms and five cases presented bilateral common iliac aneurysms. EVAR was performed in all 12 cases. Seven patients were operated under general anesthesia and five under local anesthesia. There was $100 \%$ technical success. All the postoperative angiographies showed no obvious type I or type III endoleaks, but type II endoleaks were observed in five cases. Embolization of the unilateral internal iliac artery occurred in two cases.

Bilateral CFAs were opened in eight cases. The unilateral CFA was open in two cases, and the "preclose" technique was used in the contralateral. In two cases, "preclose" was bilaterally used. Endurant was used in two cases, Talent in four cases, and Zenith in six cases. The mean operation time was $3.4 \pm 1.3 \mathrm{~h}$ (range: $2.0-4.6 \mathrm{~h}$ ). The mean bleeding volume was $150.8 \pm 60.5 \mathrm{~mL}$ (range: $75-800 \mathrm{~mL}$ ). The mean postoperative time in hospital was 8.4 \pm 2.3 days (range: $4-13$ days). There was no need for open conversion. Five patients were 
transferred to the ICU, including two emergency cases. The mean time in the ICU was 3.5 days (range: 2-7 days). The "preclose" suture technique was successfully performed at six puncture points. Three Proglides were used at one puncture point, two at three points and one at two points.

\section{Early outcomes}

One ruptured AAA patient died during the perioperative period $(8.3 \%)$. The patient presented acute respiratory distress syndrome and abdominal compartment syndrome postoperatively, and died because of multiple organ failure on the fifth day after repair. In the perioperative period, four cases presented complications. One was arrhythmia, one was left heart failure, and two were renal failure (which had been diagnosed before the repair). After drug treatment, these complications were either controlled or disappeared. Local complications presented in three cases, including leakage of the incision lymph, lung infection, and lower limb arterial embolism. The former two complications were cured after treatment. The patient with lower limb arterial embolism presented lower skin temperature of the left leg, cyanotic foot skin, and disappearance of the anterior tibial artery. After anticoagulant therapy with low molecular weight heparin calcium (Frarparine, Glaxo Smith Kline), the symptoms were relieved. There was no change in the ankle-brachial index. There was no obvious difference in age, contrast volume, repair period, or blood loss between the general anesthesia group and the local anesthesia group $(\mathrm{P}>0.05)$. However, the complication incidence of the general anesthesia group was significantly higher than that of the local anesthesia group $(\mathrm{P}=0.001)$. The perioperative mortality of the emergency repair group was higher than that of the elective repair group ( $50 \%$ vs none).

\section{Follow-up results}

The follow-up with CT angiography in 11 patients at the third month following operation revealed that the AAA diameter decreased in four cases. No other sacs were expanded. Thrombus was evident in the sac cavity. There was no combination of dissecting or pseudoaneurysm at puncture points and connective areas. Five cases showed type II endoleaks, and endoleaks disappeared in two cases. A proximal type I endoleak appeared in one case 1 year after repair, and the diameter of the AAA increased. This endoleak disappeared after the Palmaz (Cordis, US) stent was used. One case presented a distal type I endoleak 2 years after EVAR, in which the AAA diameter increased. The endoleak disappeared after the deployment of an additional iliac stent graft.

One patient died from acute myocardial infarction 9 months after repair. Three patients died from malignant tumors and heart failure 3 years after repair. Four patients died 5 years after EVAR, two from a cerebrovascular accident, and two for unknown reason. Three survivors remained for ongoing follow-up. In summary, the clinical success rate was $75 \%$ (9/12). The mortality rate was $8.3 \%$ at 30 days $(1 / 12), 16.7 \%$ at 1 year $(2 / 12), 41.7 \%(5 / 12)$ at 3 years, and $75 \%(9 / 12)$ at 5 years. The mean survival of the 11 patients who expired beyond the first 30 days was 28.5 months (range: $9-73$ months). The mean survival period after repair of the eight patients who died out of the perioperative period was 21.4 months. The survival periods after repair of three patients still alive were 73,32 , and 15 months. 


\section{DISCUSSION}

The EVAR technique is commonly chosen to prevent AAA rupture. Operative risk assessment should be performed before repair, which is particularly significant for elderly patients. To date, some AAA risk assessment models for patients who accept EVAR have been developed (Glasgow's scale and Leiden's scale) to estimate perioperative mortality (Nesi et al., 2004; Biancari et al., 2006). In these models, age is an important factor, because even though there is no other risk factor, ages of 80 years or older could be a contraindication. EVAR has been the first choice to treat AAA based on its effects on decreasing perioperative mortality, operative time, and blood loss. However, it was not clear whether these advantages also apply to nonagenarian patients. The EVAR-2 trial divided AAA patients who were intolerant to open operations into two groups: the EVAR group and the non-repair group. The results demonstrated no advantages in the EVAR group 60 years or older (EVAR Trial Participants, 2005). Nonetheless, using the same definition of risk factors, Sicard et al. (2006) demonstrated that EVAR was safe and effective for elderly patients.

With respect to octogenarian AAA patients, although their life expectancy is uncertain, several studies have reported that they show positive results to not only endovascular treatment, but also to open operations; however, only a few reports on AAA nonagenarians exist. The perioperative mortality rate was found to be $4-11 \%$ (Baas et al., 2008). In the present study, there was $100 \%$ technical success with no open-surgery conversion during the operation. One patient died in the perioperative period, which represents a mortality rate $(8.3 \%)$ that is higher than that of the DREAM trial (1.2\%) and of general octogenarian AAA patients (0.5-3.3\%). A great difference was observed in the incidence of complications between the local anesthesia group and the general anesthesia group, which was consistent with results of other reports. Goldstein et al. (2010) indicated that survival rate decreased greatly when the patient presented five or more kinds of diseases, and thus conservative treatments should be chosen in such cases. For patients under general anesthesia, the complication risks after repair increased, especially heart complications. Therefore, older patients presenting a history of coronary artery disease should think carefully before choosing general anesthesia, which was also supported in previous studies (Baril et al., 2006; Goldstein et al., 2010; Jim et al., 2010; Prenner et al., 2011). Furthermore, local anesthesia was found to be a safer and more feasible option due to the application of the new stent grafts (Endurant) and technique ("preclose").

According to results of the present study and previous reports, it is easy to achieve technical success on AAA nonagenarians. Nevertheless, attention should be drawn to the purpose of EVAR, which is to prevent complications initiated by aneurysms in order to prolong the survival period. For patients whose life expectancy is not long, technical success is not relevant. When deciding to perform the EVAR, the natural process of the disease should be weighed with respect to the life expectancy of the patient. In the USA in 2006, the mean life expectancy of nonagenarians was 4.6 years (4.1 years for females and 4.8 years for males) (National Vitals Statistics System, 2009). Although there is no related report for China, the life expectancy of different population groups is likely to be different due to regional differences and wealth gaps. A large UK trial showed that the annual rupture rate of AAA with $3.9 \mathrm{~cm}$ in diameter was $0.3 \%$, those with $4.0-4.9 \mathrm{~cm}$ in diameter was $1.5 \%$, and those with $5.0-5.9 \mathrm{~cm}$ in diameter was $0.6 \%$. Therefore, surgical intervention was suggested for sacs with diameters of 4.5-5.5 $\mathrm{cm}$ or above, which increased by $5 \mathrm{~mm}$ six months later (Jones et al., 1998; Brady 
et al., 2004). Jones et al. (1998) evaluated 57 cases of AAA patients who were not suitable for repair. The mean age of the patients was 81 . In the follow-up period of 2 years, 50 patients died, $38 \%$ of which were due to AAA rupture. The mean survival period of patients with AAA diameters of 5.0-5.9 $\mathrm{cm}$ or above was 21 months, whereas that of patients with AAA diameters more than $6.0 \mathrm{~cm}$ was 15 months. Conway et al. (2001) reported the results of a 10 -year follow-up of 106 patients who were unable to accept AAA operations. The mean age was 78.4 years. Seventy-six patients died, $49 \%$ of which were due to AAA rupture. The mean survival period after repair was 17 months. The survival rates of 1 year, 2 years, and 3 years were 54,40 , and $17 \%$, respectively. Therefore, compared with the survival period of patients that did not undergo the AAA operation, the survival period of older patients in our study was not prolonged, which demonstrates the uncertainty of whether or not to perform EVAR on older patients. Nonetheless, the efficacy of EVAR on older patients cannot be denied. For the symptomatic patients and patients presenting risk of rupture or with rupture, EVAR is essentially the only option. For asymptomatic patients, if complications could be controlled to a small extent, EVAR is a reasonable treatment. Additionally, the psychological impact of the repair procedure in patients is obvious. Patients were relieved from mental pressure after the operation. Further study is still necessary in order to establish to what degree such psychological suggestion can work. In addition, the willingness of family members is also an important factor of the choice.

The present study is retrospective. Thus, the elderly AAA patients who came to our hospital generally showed good health and economic conditions, which resulted in good treatment results. However, some questions persist. It remains unknown whether the AAA of these elderly patients is similar to the disease in younger patients for which the process develops slowly and is not easy to rupture. Furthermore, little is known about the natural history of the disease in nonagenarians.

\section{CONCLUSIONS}

For AAA patients aged 90 years and above, EVAR was found to be safe with low perioperative morbidity and mortality rates. The medium and long-term results suggested that EVAR may be of limited benefit in some patients who are aged $>90$ years. Therefore, individual patient selection is very important. Elderly AAA patients benefited from the decreased risks of EVAR from applications of the total puncture technique and new stent-graft delivery systems.

\section{REFERENCES}

Baas AF, Janssen KJ, Prinssen M, Buskens E, et al. (2008). The Glasgow Aneurysm Score as a tool to predict 30-day and 2-year mortality in the patients from the Dutch Randomized Endovascular Aneurysm Management trial. J. Vasc. Surg. 47: 277-281.

Baril DT, Palchik E, Carroccio A, Olin JW, et al. (2006). Experience with endovascular abdominal aortic aneurysm repair in nonagenarians. J. Endovasc. Ther. 13: 330-337.

Biancari F, Hobo R and Juvonen T (2006). Glasgow Aneurysm Score predicts survival after endovascular stenting of abdominal aortic aneurysm in patients from the EUROSTAR registry. Br. J. Surg. 93: 191-194.

Brady AR, Thompson SG, Fowkes FG, Greenhalgh RM, et al. (2004). Abdominal aortic aneurysm expansion: risk factors and time intervals for surveillance. Circulation 110: 16-21.

Brinkman WT, Terramani TT, Najibi S, Weiss VJ, et al. (2004). Endovascular abdominal aortic aneurysm repair in the octogenarian. Ann. Vasc. Surg. 18: 401-407. 
Conway KP, Byrne J, Townsend M and Lane IF (2001). Prognosis of patients turned down for conventional abdominal aortic aneurysm repair in the endovascular and sonographic era: Szilagyi revisited? J. Vasc. Surg. 33: 752-757.

Dosluoglu HH, Cherr GS, Harris LM and Dryjski ML (2007). Total percutaneous endovascular repair of abdominal aortic aneurysms using Perclose ProGlide closure devices. J. Endovasc. Ther. 14: 184-188.

EVAR Trial Participants (2005). Endovascular aneurysm repair and outcome in patients unfit for open repair of abdominal aortic aneurysm (EVAR trial 2): randomised controlled trial. Lancet 365: 2187-2192.

Goldstein LJ, Halpern JA, Rezayat C, Gallagher KA, et al. (2010). Endovascular aneurysm repair in nonagenarians is safe and effective. J. Vasc. Surg. 52: 1140-1146.

Haug ES, Romundstad P, Aune S, Hayes TB, et al. (2005). Elective open operation for abdominal aortic aneurysm in octogenarians - survival analysis of 105 patients. Eur. J. Vasc. Endovasc. Surg. 29: 489-495.

Henebiens M, Vahl A and Koelemay MJ (2008). Elective surgery of abdominal aortic aneurysms in octogenarians: a systematic review. J. Vasc. Surg. 47: 676-681.

Jim J, Sanchez LA, Sicard GA, Curci JA, et al. (2010). Acceptable risk but small benefit of endovascular aneurysm repair in nonagenarians. Ann. Vasc. Surg. 24: 441-446.

Jones A, Cahill D and Gardham R (1998). Outcome in patients with a large abdominal aortic aneurysm considered unfit for surgery. Br. J. Surg. 85: 1382-1384.

Lange C, Leurs LJ, Buth J and Myhre HO (2005). Endovascular repair of abdominal aortic aneurysm in octogenarians: an analysis based on EUROSTAR data. J. Vasc. Surg. 42: 624-630.

National Vitals Statistics System (2009). Available at [www.cdc.gov/nchs/death.htm]. Accessed July 1, 2009.

Nesi F, Leo E, Biancari F, Bartolucci R, et al. (2004). Preoperative risk stratification in patients undergoing elective infrarenal aortic aneurysm surgery: evaluation of five risk scoring methods. Eur. J. Vasc. Endovasc. Surg. 28: 52-58.

Prenner SB, Turnbull IC, Serrao GW, Fishman E, et al. (2011). Outcome of elective endovascular abdominal aortic aneurysm repair in nonagenarians. J. Vasc. Surg. 54: 287-294.

Sicard GA, Zwolak RM, Sidawy AN, White RA, et al. (2006). Endovascular abdominal aortic aneurysm repair: long-term outcome measures in patients at high-risk for open surgery. J. Vasc. Surg. 44: 229-236.

U.S. Census Bureau (2009). Available at [www.census.gov/population/www/projections/index.html]. Accessed July 1, 2009. 